\title{
EFECTO DE LA AGREGACIÓN DE UNIVERSIDADES ESPAÑOLAS EN EL RANKING DE SHANGHAI (ARWU): CASO DE LAS COMUNIDADES AUTÓNOMAS Y LOS CAMPUS DE EXCELENCIA
}

\author{
Domingo Docampo, Francisco Herrera, Teodoro Luque-Martínez \\ y Daniel Torres-Salinas
}

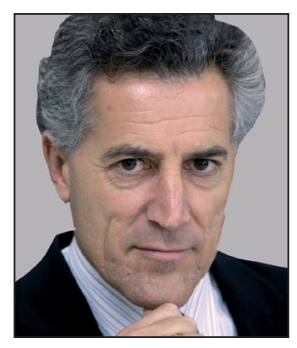

Domingo Docampo es catedrático en la Universidad de Vigo, de la que fue rector entre los años 1998 y 2006 . Se licenció en matemáticas en 1976 en la Universidad de Valladolid, y obtuvo el grado de doctor en matemáticas por la Universidad de Santiago de Compostela en 1984. Es autor de un centenar de trabajos de investigación en el ámbito de la teoría de la señal y las comunicaciones. Desde hace unos años centra parte de su esfuerzo investigador en el análisis internacional comparado de instituciones universitarias.

Universidade de Vigo Atlantic Research Center for Information and Communication Technologies Campus Universitario, 36310 Vigo, España ddocampo@uvigo.es

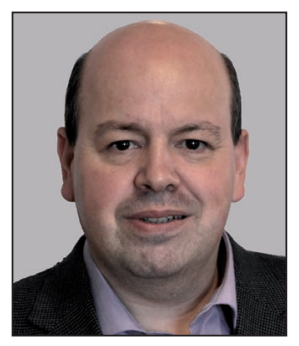

Francisco Herrera es catedrático del departamento Ciencias de la Computación e Inteligencia Artificial de la Universidad de Granada, y director del grupo de investigación Soft Computing y Sistemas de Información Inteligentes. Ha dirigido 25 tesis doctorales y publicado más de 200 artículos en revistas internacionales en las áreas de inteligencia computacional, minería de datos y bibliometría. Es co-creador de los 'Rankings I-UGR de universidades según campos y disciplinas científicas'.

Universidad de Granada Depto. de Ciencias de la Computación e Inteligencia Artificial, Etsiit, 18071 Granada, España herrera@decsai.ugr.es

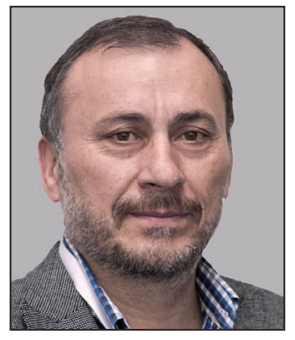

Teodoro Luque-Martínez es catedrático de la Universidad de Granada, y director del grupo de investigación Administración de Empresas y Marketing (Ademar). Ha dirigido 9 tesis doctorales, autor de 17 libros, 50 artículos científicos y director de numerosos proyectos en colaboración con empresas e instituciones públicas y privadas. Interesado en la planificación estratégica y gestión de universidades y del estudio de su impacto en el territorio. Actualmente es coordinador del CEI BioTic Granada.

Universidad de Granada Grupo de investigación Administración de Empresas y Marketing Campus Cartuja, 18071, Granada, España tluque@ugr.es

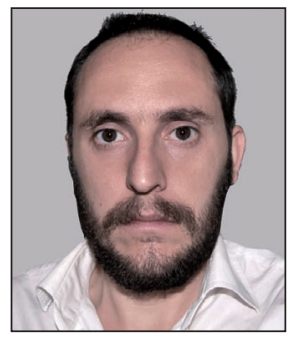

Daniel Torres-Salinas es doctor en documentación científica y trabaja como técnico de gestión de la investigación en la Universidad de Navarra, donde realiza auditorías sobre la calidad de la investigación desde una perspectiva bibliométrica, asimismo pertenece al grupo EC3 de la Universidad de Granada. Es miembro del think tank ThinkEPI y co-creador de los 'Rankings I-UGR de universidades según campos y disciplinas científicas'.

Universidad de Navarra, Grupo Evaluación de la Ciencia y la Comunicación Científica Centro de Investigación Médica Aplicada Pío XII, 55. 31008 Pamplona, España torressalinas@gmail.com

\section{Resumen}

El Academic Ranking of World Universities (ARWU) o Ranking de Shanghai es actualmente el referente para conocer la posición de las universidades a nivel mundial. La escalada de puestos en dicho ranking se ha convertido en el objetivo de universidades y gobiernos. No es extraño por tanto que se haya planteado en España la posibilidad de sugerir agregaciones de universidades con el fin de subir puestos. En ese contexto, el objetivo del presente trabajo es determinar la posición que ocuparían en ARWU las comunidades autónomas españolas y los Campus de excelencia internacional (CEI) si constituyeran una única universidad. Para calcular los indicadores de ARWU para esas posibles agregaciones se sigue el trabajo de Docampo (2012b). Los resultados muestran con claridad la dificultad para ascender puestos pese a la suma de instituciones. Tan sólo agregando todas las universidades catalanas se podría situar una universidad española en el top 100. De los CEls, los puestos más relevantes los ocuparían Habitat $5 U$ (universidades de la Comunidad Valenciana), BKC (Barcelona y Politècnica de Catalunya) y VLC/Campus (Valencia y Politécnica de Valencia) que estarían en el rango 150-200.

Artículo recibido el 19-04-12 


\title{
Palabras clave
}

Rankings de universidades, Ranking de Shanghai, ARWU, Campus de excelencia internacional, CEI, Política científica, España, Educación superior, Indicadores bibliométricos.

Title: Aggregate ranking of Spain's universities in the Shanghai Ranking (ARWU):

Effect on autonomous communities and campuses of international excellence

\begin{abstract}
The Academic Ranking of World Universities ( $A R W U$ ) or Shanghai Ranking is a de facto standard to determine the position of universities worldwide. Improving ranking positions has become the goal of universities and governments. No wonder therefore that the aggregation of universities in Spain is nowadays suggested as a means to raise the international profile of our university system. In this context, the goal of this study was to test two potential groupings, universities belonging to the same Autonomous Community and campuses of international excellence (CEIs), to determine the ARWU placement that would result. To compute the ARWU indicators of these possible groupings, we used the methodology developed in Docampo (2012b). The results of our analysis clearly show the difficulty of improving ranking positions by calculating the sum of aggregated institutions. As a matter of fact, the only way to place a university cluster from Spain in the top 100 would be to treat all of the Catalan universities as a single institution. The next best ranking for Spain would be achieved by the Habitat $5 U$ (universities of Comunidad Valenciana), BKC (Barcelona and Politècnica de Catalunya) and VLC/Campus (Valencia and Politécnica de Valencia), CEls which would lie in the 150-200 range.
\end{abstract}

\section{Keywords}

University rankings, Shanghai Ranking, $A R W U$, International excellence campus, Scientific policy, Spain, Higher education, Bibliometrics indicators.

Docampo, Domingo; Herrera, Francisco; Luque-Martínez, Teodoro; Torres-Salinas, Daniel. “Efecto de la agregación de universidades españolas en el Ranking de Shanghai (ARWU): caso de las comunidades autónomas y los campus de excelencia". El profesional de la información, 2012, julio-agosto, v. 21, n. 4, pp. 428-432.

http://dx.doi.org/10.3145/epi.2012.jul.16

\section{Introducción}

En 2003 se publicó por vez primera el Shanghai Jiao Tong Academic Ranking of World Universities (ARWU) conocido como Ranking de Shanghai. Desde entonces, pese a la proliferación de otros rankings de universidades (Torres-Salinas et al., 2011) y pese a las críticas recibidas desde sus inicios (Van Raan, 2005), ARWU se ha convertido en el referente básico para conocer la posición de las universidades a nivel mundial. Actualmente uno de los objetivos de gobiernos y universidades es escalar puestos en $A R W U$ que además es utilizado para tomar todo tipo de decisiones desde la distribución de fondos hasta la selección de un centro para una estancia de investigación (Delgado, 2012). Así pues, la posición de una universidad en este ranking se está convirtiendo en un factor clave para su futuro más inmediato, y puede ser determinante en el éxito o fracaso de muchas estrategias e iniciativas. Una de las más duras críticas que está sufriendo el sistema universitario español es la mala situación de sus universidades ya que ninguna de ellas logra situarse en el top 100 y ni tan siquiera en el top 150; solamente a partir del rango de posiciones 201-300 comenzamos a encontrar alguna institución española.

Se ha señalado que el sistema universitario español está aquejado, entre otros males, de estar compuesto por un excesivo número de universidades, y se sugiere la necesidad de fusión de las mismas como un medio para fortalecer internacionalmente la posición del sistema. Una de las convocatorias más controvertidas de los últimos

\section{El Ranking de Shanghai se ha convertido en un referente básico para conocer la posición de las universidades y todos in- tentan escalar puestos en el mismo}

años ha sido la de los Campus de excelencia internacional (CEIs), dirigida a conseguir campus más competitivos y situarlos entre los de más prestigio internacional. Los CEls son proyectos liderados por universidades en los que se contemplaba la agregación con otras instituciones (Luque, 2011), pero muy especialmente entre universidades, lo que supuestamente debería implicar también la subida de puestos en $A R W U$. Por tanto, si tuviésemos en cuenta las agregaciones universitarias de los CEls es lógico preguntarse por la posición que ocuparían éstas en el Ranking de Shanghai con el fin de aportar evidencias para el debate que ya ha comenzado subrepticiamente en la sociedad. Hasta hace bien poco esta pregunta era difícil de responder ya que pese a disponer de los criterios de confección era generalizado el desconocimiento acerca de los cálculos y operaciones matemáticas que se llevaban a cabo en el $A R W U$. De esta forma era casi imposible reproducir los resultados del ranking. Sin embargo, las recientes publicaciones de uno de los autores han desvelado con exactitud cómo replicar dichos cálculos, por lo que estamos ya en disposición de poder estimar y predecir con un alto nivel de certeza sus resultados (Docampo, 2012b). 
Teniendo en cuenta el debate sobre la fusión de universidades, la puesta en marcha de los CEls y la reproducibilidad actual del $A R W U$, el objetivo de este artículo es estudiar los efectos de las agregaciones de universidades españolas en relación con su escalada de posiciones en dicho ranking en dos simulaciones que responderían a las siguientes interrogantes:

1) ¿Cuál sería la posición de cada una de las comunidades autónomas en el supuesto de que todas sus universidades se fusionaran creando una única universidad por comunidad?

2) ¿Qué posición ocuparían los diversos CEls si las universidades que los componen se fusionaran en una única universidad?

Se ha señalado que uno de los problemas del sistema universitario español es la existencia de un excesivo número de universidades, por lo que se sugiere su fusión

\section{Material y métodos}

El Ranking de Shanghai se basa en:

- análisis de datos bibliométricos sobre el número de premios relevantes (premios Nobel, medallas Field) obtenidos por el alumnado y el profesorado de las universidades para generar sus dos primeros indicadores (Alumni y Awards);

- investigadores más citados en 21 áreas de investigación determinadas por Thomson Reuters (indicador HiCi);

- artículos publicados en Nature y Science en los últimos cinco años (indicador N\&S);
- registrados en el Science Citation Index Expanded (SCIE) y el Social Science Citation Index (SSCl) en el año anterior a la publicación del ranking, que producen el quinto indicador (PUB); y

- en el caso de una serie de países, entre los que se encuentra España, la prestación relativa en función de las dimensiones de cada institución, lo que da lugar al sexto indicador $(P C P)$.

Fusionando todas las universidades de cada comunidad sólo Cataluña se situaría entre las 100 primeras

Así pues, los cinco primeros son directos, mientras que el sexto es compuesto: se basa en los cinco anteriores y el tamaño de la universidad. Una completa descripción de los indicadores y la metodología utilizada en la elaboración del ranking puede encontrarse en Liu y Cheng (2005).

Por otro lado, como hemos comentado en una serie de artículos que se inician en 2008 (Docampo, 2008, 2011, 2012a) en los que se analizan los resultados del ranking a nivel de sistemas universitarios, se consiguió averiguar cómo calcular alguno de los indicadores, buscando una proporcionalidad no lineal para el cálculo final del indicador. Esa investigación ha llegado a su final y en Docampo (2012b) se ha mostrado con exactitud cómo reproducir los resultados de $A R W U$. Teniendo como base ese trabajo y teniendo como población de estudio a las universidades españolas, se han calculado para cada una de ellas todos los indicadores del Shanghai en la próxima edición de 2012 que se publicará en agosto. Posteriormente los resultados de las universidades se han agregado para los niveles establecidos en los objetivos, comunidades autónomas y CEls, determinándose el

\begin{tabular}{|c|c|c|c|c|c|c|c|c|}
\hline \multirow[b]{2}{*}{ Comunidad autónoma } & \multicolumn{6}{|c|}{ INDICADORES ARWU } & \multirow{2}{*}{$\begin{array}{l}\text { Total } \\
\text { puntos }\end{array}$} & \multirow{2}{*}{$\begin{array}{c}\text { ARWU } 2012 \\
\text { Posición top } 500\end{array}$} \\
\hline & Alumni & Award & $\mathrm{HiCi}$ & N\&S & PUB & $P C P$ & & \\
\hline Universidades de Cataluña & 0 & 0 & 10,2 & 24,5 & 81,4 & 15,8 & 25,5 & $85-90$ \\
\hline Universidades de Madrid & 19 & 0 & 12,5 & 18,7 & 70,4 & 11,6 & 24,0 & $105-110$ \\
\hline Universidades de la Com. Valenciana & 0 & 0 & 8,9 & 17,3 & 60,3 & 13,0 & 19,1 & \multirow{2}{*}{$150-200$} \\
\hline Universidades de Andalucía & 0 & 0 & 0 & 13,7 & 66,0 & 10,6 & 17,5 & \\
\hline Universidades de Galicia & 0 & 0 & 7,2 & 6,9 & 43,6 & 11,9 & 13,1 & \multirow{3}{*}{$300-400$} \\
\hline Universidad del País Vasco & 0 & 0 & 0 & 8,1 & 41,6 & 13,1 & 11,6 & \\
\hline Universidades de Castilla y León & 0 & 0 & 7,2 & 4,9 & 36,8 & 9,8 & 11,1 & \\
\hline Universidad de Zaragoza & 0 & 0 & 5,1 & 1,5 & 33,8 & 12,3 & 9,6 & $400-500$ \\
\hline Universidad de Oviedo & 0 & 0 & 0 & 10,9 & 27,3 & 9,8 & 8,8 & \multirow{9}{*}{ Fuera } \\
\hline Universidades de Canarias & 0 & 0 & 0 & 7,4 & 27,9 & 11,0 & 8,4 & \\
\hline Universidades de la Región de Murcia & 0 & 0 & 0 & 1,5 & 30,0 & 11,5 & 7,7 & \\
\hline Universidad de Castilla La Mancha & 0 & 0 & 0 & 4,8 & 25,6 & 13,5 & 7,6 & \\
\hline Universidad de Cantabria & 0 & 0 & 0 & 7,3 & 21,5 & 14,1 & 7,4 & \\
\hline Universidades de la Com. F. de Navarra & 0 & 0 & 0 & 0 & 28,4 & 13,9 & 7,3 & \\
\hline Universitat de les Illes Balears & 0 & 0 & 5,1 & 3,7 & 19,2 & 13,8 & 7,2 & \\
\hline Universidad de Extremadura & 0 & 0 & 0 & 6,9 & 13,2 & 11,8 & 5,4 & \\
\hline Universidad de La Rioja & 0 & 0 & 0 & 0 & 11,1 & 10,8 & 3,4 & \\
\hline
\end{tabular}

Tabla 1. Posición de las universidades españolas agregadas por comunidad autónoma para la edición 2012 del Ranking de Shanghai 


\begin{tabular}{|c|c|c|c|c|c|c|c|c|c|}
\hline \multirow[b]{2}{*}{ Acrónimo CEI } & \multirow[b]{2}{*}{ Universidades integrantes } & \multicolumn{6}{|c|}{ INDICADORES ARWU } & \multirow{2}{*}{$\begin{array}{l}\text { Total } \\
\text { puntos }\end{array}$} & \multirow{2}{*}{$\begin{array}{c}\text { ARWU } 2012 \\
\text { Posición top } 500\end{array}$} \\
\hline & & Alumni & Award & $\mathrm{HiCi}$ & $N \& S$ & PUB & $P C P$ & & \\
\hline CEI-Habitat $5 U$ & UV-UPV-UII-UMH-UAL & 0 & 0 & 8,9 & 16,9 & 60,3 & 13,3 & 19,1 & \multirow{3}{*}{$150-200$} \\
\hline$B K C$ & $U B-U P C$ & 0 & 0 & 10,2 & 12,8 & 56,9 & 14,8 & 17,9 & \\
\hline VLC/Campus & UV-UPV & 0 & 0 & 8,9 & 15,2 & 50,5 & 13,3 & 16,7 & \\
\hline Campus Moncloa & UCM-UPM & 19 & 0 & 0 & 10,4 & 51,7 & 10,7 & 15,8 & \multirow{5}{*}{$200-300$} \\
\hline$H U B$ & $U B$ & 0 & 0 & 10,2 & 10,2 & 47,5 & 15,3 & 15,5 & \\
\hline Campus UAM/CSIC & UAM & 0 & 0 & 12,5 & 13,5 & 39,0 & 17,1 & 15,1 & \\
\hline UABCEI & $U A B$ & 0 & 0 & 0 & 12,4 & 44,8 & 17,7 & 13,6 & \\
\hline Ceimar & UGR-UALM-UCA-UMA-UHU & 0 & 0 & 0 & 10,5 & 49,9 & 10,5 & 13,5 & \\
\hline Campus do Mar & UDC-USC-UVIGO & 0 & 0 & 7,2 & 6,9 & 43,6 & 11,9 & 13,1 & \multirow{6}{*}{$300-400$} \\
\hline CEI-Energintel & UMU-UPCT-UAH-URJC-UEX & 0 & 0 & 0 & 6,9 & 45,2 & 11,1 & 11,8 & \\
\hline Euskampus & EHU/UPV & 0 & 0 & 7,2 & 9,9 & 33,9 & 12,1 & 11,7 & \\
\hline CEl-lberus & UNIZAR-UNIRIOJA-UDL-UPN & 0 & 0 & 5,1 & 1,5 & 41,8 & 12,1 & 11,2 & \\
\hline Biotic Granada & UGR & 0 & 0 & 0 & 8,5 & 39,0 & 12,3 & 11,0 & \\
\hline Icaria INT & UPF & 0 & 0 & 0 & 15,6 & 24,6 & 26,2 & 10,9 & \\
\hline Andalucía Tech & UMA-USE & 0 & 0 & 0 & 8,1 & 39,1 & 9,9 & 10,7 & \multirow{3}{*}{$400-500$} \\
\hline CEI-A3 & UCA-UCO-UALM-UHU-UJaén & 0 & 0 & 0 & 7,1 & 39,0 & 11,0 & 10,6 & \\
\hline Campus Energía & $U P C$ & 0 & 0 & 0 & 7,7 & 32,4 & 14,1 & 9,7 & \\
\hline Campus Vida & USC & 0 & 0 & 0 & 6,1 & 31,8 & 12,1 & 9,0 & \multirow{15}{*}{ Fuera } \\
\hline CEI-Turiagua & UIB-UDG & 0 & 0 & 5,1 & 3,7 & 27,4 & 14,1 & 8,9 & \\
\hline Atlántico Tricontinental & ULL-ULPGC & 0 & 0 & 0 & 8,5 & 29,2 & 10,2 & 8,8 & \\
\hline Horizontes del Hombre & UVA-UBU-ULEON & 0 & 0 & 7,2 & 2,1 & 28,3 & 9,5 & 8,7 & \\
\hline AD Futurum & UNIOVI & 0 & 0 & 0 & 7,4 & 27,9 & 11,0 & 8,4 & \\
\hline Montegancedo & UPM & 0 & 0 & 0 & 4,9 & 29,8 & 10,0 & 8,2 & \\
\hline Catalunya Sud & URV & 0 & 0 & 0 & 6,2 & 23,4 & 17,7 & 7,9 & \\
\hline Mare Nostrum 37/38 & UMU-UPCT & 0 & 0 & 0 & 1,5 & 30,0 & 11,5 & 7,7 & \\
\hline Cytema & UCLM & 0 & 0 & 0 & 5,0 & 25,6 & 13,5 & 7,7 & \\
\hline Cantabria CEI & UNICAN-UIMP & 0 & 0 & 0 & 7,6 & 21,5 & 14,2 & 7,4 & \\
\hline Studi Salmantini & USAL & 0 & 0 & 0 & 4,7 & 24,2 & 10,4 & 7,0 & \\
\hline Horizonte 2015 & UNA & 0 & 0 & 0 & 0 & 23,2 & 15,0 & 6,3 & \\
\hline Carlos III 2011 & UC3M & 0 & 0 & 0 & 0 & 23,1 & 13,0 & 6,1 & \\
\hline Hidranatura & UEX & 0 & 0 & 0 & 0 & 20,4 & 10,5 & 5,3 & \\
\hline Aristos & UDEUSTO-COMILLAS-URLL & 0 & 0 & 0 & 0 & 14,4 & 8,7 & 3,9 & \\
\hline
\end{tabular}

Tabla 2. Posición de las universidades españolas agregadas por CEl para la edición 2012 del Ranking de Shanghai

rango aproximado que ocuparían. El material complementario a este estudio puede encontrarse en la web: http://www.rankinguniversidades.es/shanghai

Si las universidades estuvieran agregadas según las alianzas CEI tendríamos 17 en el top 500 del Ranking de Shanghai (al menos se reducirían en 3 evitando duplicidades) aunque ninguno en el top 100

\section{Resultados}

En la edición 2011 del Ranking de Shanghai estuvieron presentes once universidades españolas. Cinco aparecieron entre los puestos 401-500 (Granada, Pompeu Fabra, Santiago,
Vigo y Zaragoza) dos en el rango 301-400 (Autónoma de Barcelona y Politécnica de Valencia) y cuatro entre los puestos 201-300 (Autónoma de Madrid, Barcelona, Complutense y Valencia). En la tabla 1 presentamos cómo se comportarían las universidades si se agregaran por comunidad autónoma. Nueve comunidades, las de menor tamaño o aquellas que sólo cuentan con una o dos universidades, no entrarían entre las quinientas primeras. Las ocho comunidades restantes si entrarían entre las 500 primeras universidades. Destaca Cataluña que se sitúa entre las 100 primeras, en torno a la posición 85-90. Le seguiría la Comunidad de Madrid, ya fuera del top 100, que se colocaría en el rango 105-110. El resto de comunidades las localizaríamos a partir de la posición 150. Más concretamente la Comunidad Valenciana y Andalucía entre las 150-200, Galicia (300-400), País Vasco (300-400), Castilla y León (300-400) y Aragón (400-500). Esto significa que a pesar de sumar todas las universidades por comunidad a duras penas alcanzaríamos a emplazar una 
única universidad en el top 100. Esta situación evidencia la dificultad para escalar puestos en el ARWU.

En relación con los CEls (tabla 2) si las universidades estuvieran agregadas en este sentido tendríamos $17 \mathrm{CEI}$ en $A R W U$ teniendo en cuenta que algunas de las universidades pueden computar en varios CEIs al mismo tiempo y por tanto el resultado sería menor (como mínimo se reduciría en 3, porque UB, UPC y UGR deberían optar por un CEI). Ninguno de los CEls lograría estar entre los 100 primeros puestos. Los mejores resultados los alcanzan Habitat $5 U$ (universidades de la Comunidad Valenciana), BKC (Barcelona y Politècnica de Catalunya) y VLC/Campus (Valencia y Politécnica de Valencia) que se colocarían en la horquilla 150-200. Éstos resultados no suponen una mejora sustancial de los puestos que ya ocupan a nivel individual universidades como Barcelona, Valencia o Politécnica de Valencia. Una situación similar ocurre con el Campus Moncloa (Complutense y Politécnica de Madrid) que se colocaría entre los puestos 200300 , una posición similar a la ya ocupada por la Complutense. Otras agregaciones sí tendrían algún efecto más positivo como por ejemplo Campus do Mar (A Coruña, Santiago de Compostela y Vigo) que estaría entre las 300-400, muy cercana a 300, mientras que en la actualidad Santiago de Compostela y Vigo están en el puesto 401-500. Sin embargo no siempre se produce este efecto tan positivo, como es el caso del CEl-Iberus (Zaragoza, Rioja, Lleida, Pública de Navarra) que ocuparía el rango 385-395 mientras que Zaragoza en solitario ya está ahora en el rango 401-500.

Los resultados del estudio aconsejan estudiar la fusión de universidades caso por caso y sobre bases diferentes a las que hasta la fecha se han establecido, y desde luego no para escalar puestos

\section{Reflexión final}

En este trabajo hemos analizado la posición en el Ranking de Shanghai de las universidades españolas si se agregaran por comunidades autónomas o por campus de excelencia. Los resultados no dejan de ser sorprendentes, ya que solamente sumando a todas las universidades catalanas conseguiríamos situar una institución española entre las 100 primeras. El análisis resta fuerza a las opiniones que sustentan la fusión de universidades ya que se comprueba la dificultad para ascender en las posiciones del ranking mediante la agregación de instituciones sin considerar otros aspectos, lo que revela que esa no es la solución correcta para escalar puestos. Esta situación ha quedado especialmente en evidencia con los CEls, que no mejorarían sustancial ni espectacularmente los resultados que ya obtienen las universidades a nivel individual. Este trabajo aporta evidencias útiles a los gestores de políticas científicas que pretendan promover es- trategias acordes con los objetivos que se hayan propuesto; en particular, los resultados del trabajo aconsejan la necesidad de estudiar la fusión de universidades caso por caso y sobre bases diferentes a las que hasta la fecha se han establecido, y desde luego con argumentos diferentes al de la posible escalada de puestos que se pudiera producir en el ranking internacional con más notoriedad.

\section{Bibliografía}

Delgado-López-Cózar, Emilio. "Cómo se cocinan los rankings universitarios". Dendra médica. Revista de humanidades, 2012, v. 11, n. 1, pp. 43-58.

http://sci2s.ugr.es/rankinguniversidades/prensa/2012/ scl03.pdf

Docampo, Domingo. "Rankings internacionales y calidad institucional". Revista de educación, 2008, № extraordinario, pp. 149-176.

http://www.revistaeducacion.mec.es/re2008/re2008_ 07.pdf

Docampo, Domingo. "On using the Shanghai ranking to assess the research performance of university systems". Scientometrics, 2011, v. 86, n. 1, pp. 77-92. http://dx.doi.org/10.1007/s11192-010-0280-y

Docampo, Domingo. "Adjusted sum of institutional scores as an indicator of the presence of university systems in the ARWU ranking". Scientometrics, 2012a, v. 90, n. 2, pp. 701713.

http://dx.doi.org/10.1007/s11192-011-0490-y

Docampo, Domingo. "Reproducibility of the Shanghai academic ranking of world universities results". Scientometrics, 2012b (en prensa).

http://dx.doi.org/10.1007/s11192-012-0801-y

Liu, Nian-Cai; Cheng, Ying. "Academic ranking of world universities: Methodologies and problems". Higher education in Europe, 2005, v. 30, n. 2, pp. 127-136. http://dx.doi.org/10.1080/03797720500260116

Luque-Martínez, Teodoro. "University and development: A shared commitment". En: Marketing akademicki Rola Uniwersytetów w Promocji Miast i Regionów, Lodz (Poland), 2011.

Torres-Salinas, Daniel; Delgado-López-Cózar, Emilio; García-Moreno-Torres, José; Herrera, Francisco. "Rankings ISI de las universidades españolas por campos científicos: Descripción y resultados". El profesional de la información, 2011 , v. 20, n. 1, pp. 111-112.

http://dx.doi.org/10.3145/epi.2011.nov.16

Van Raan, Anthony FJ. "Fatal attraction: conceptual and methodological problems in the ranking of universities by bibliometric methods". Scientometrics, 2005, v. 62, n. 1, pp. 133-143.

http://dx.doi.org/10.1007/s11192-005-0008-6 